\title{
Astrometric performance of the Schmidt telescope at the Xuyi station of the Purple Mountain Observatory $\dagger$
}

\author{
Y. Yu ${ }^{1}$, Z. H. Tang ${ }^{1}$, Z. X. $\mathrm{Qi}^{1,2}$ and J. F. Wu ${ }^{3}$ \\ ${ }^{1}$ Shanghai Astronomical Observatory, Chinese Academy of Sciences, Shanghai 200030, China \\ email: yuy@shao.ac.cn \\ ${ }^{2}$ Graduate School of the Chinese Academy of Sciences, Beijing 100039, China \\ ${ }^{3}$ Surveying and Mapping Institute, Zhengzhou 450052, China
}

\begin{abstract}
On January 26, 2006, an area of the sky of $80 \mathrm{deg}^{2}$ at $\alpha=9 h, \delta=20^{\circ}$ (J2000.0) was observed with the $100 / 120 \mathrm{~cm}$ Schmidt telescope at the Xuyi station of the Purple Mountain Observatory. Astrometric performance of the telescope is analyzed by this group of CCD observations. The results show that: (1) the CCD images suffer from complicated distortions, and a third order plate model is recommended for reductions; (2) there is no obvious magnitude equation for objects brighter than $16.5 \mathrm{mag}$, and (3) for this group of CCD images, the astrometric precision for objects brighter than 16.5 mag is better than 70 mas per coordinate when reduced against the $\mathrm{UCAC} 2$ catalog.
\end{abstract}

Keywords. astrometry, methods: data analysis

\section{Introduction}

The 100/120 cm Schmidt telescope at the Xuyi station of the Purple Mountain Observatory is mainly used to detect and study the Near-Earth asteroids. The focal length of the telescope is $1.8 \mathrm{~m}$, and the CCD field of view (FoV) is $2 \times 2 \mathrm{deg}^{2}$ with $4096 \times$ 4096 pixels ( 1 pixel $=15$ microns).

On January 26, 2006, an area of the sky of $80 \mathrm{deg}^{2}$ at $\alpha=9 h, \delta=20^{\circ}$ (J2000.0) was observed. Altogether $46 \mathrm{CCD}$ images were obtained. The exposure time was set at 40 seconds, and the limiting magnitude is about $18.5 \mathrm{mag}$. The FWHM of the objects is about 2-3 pixels. The UCAC2 catalog (Zacharias et al. 2004) is used as a reference. There are about 1800 reference stars on each CCD image. The measured coordinates of objects are obtained by the method of Gaussian contour fitting.

\section{Investigation of astrometric performance}

Classical CCD adjustment is performed with the 1-5 order model against the UCAC2 catalog. The potentially poor stars are eliminated if the post-fit residuals are larger than $3 \sigma$. In the case of a 2 nd-order model, the standard deviation is about 160 mas, but for the 3rd-order model, it is improved to about 75 mas and that is equivalent to those with higher order models. This precision is also close to the combined error of the reference catalog and measurements. The residual vector distribution, when using a 3rd-order model, is shown in Fig.1(a). The residuals are distributed randomly, indicating that the

$\dagger$ Supported by the National Natural Science Foundation of China(Grant Nos. 10673026, 10333050, and 10573018) and Science \& Technology Commission of Shanghai Municipality(06DZ22101). 


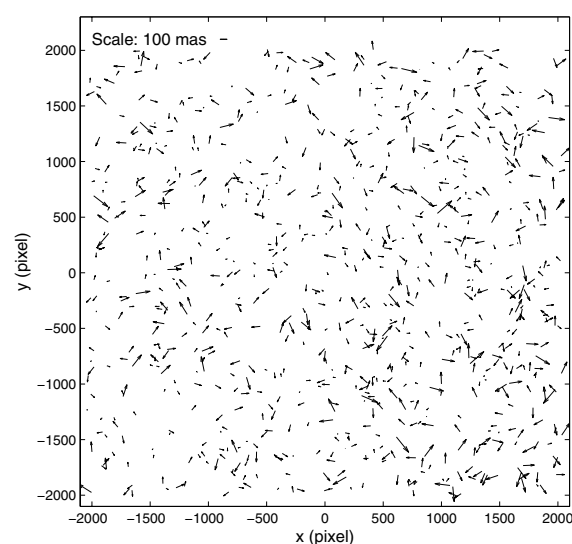

(a)

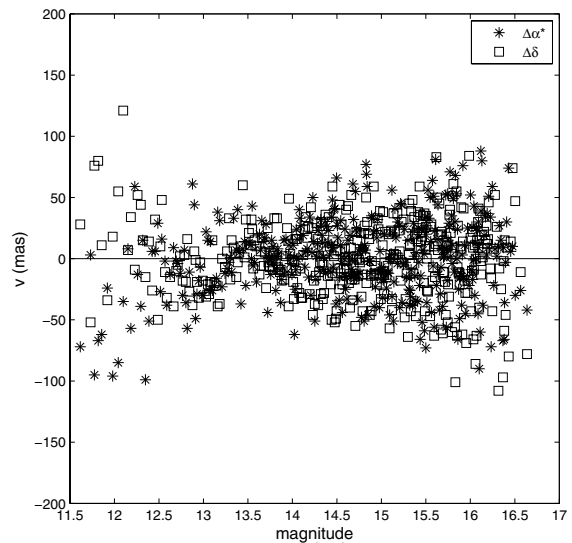

(b)

Figure 1. (a) Residual vectors distribution on images reduced with the 3 order plate model;

(b)Relationship between the residuals and magnitudes. One point represents 5 stars.

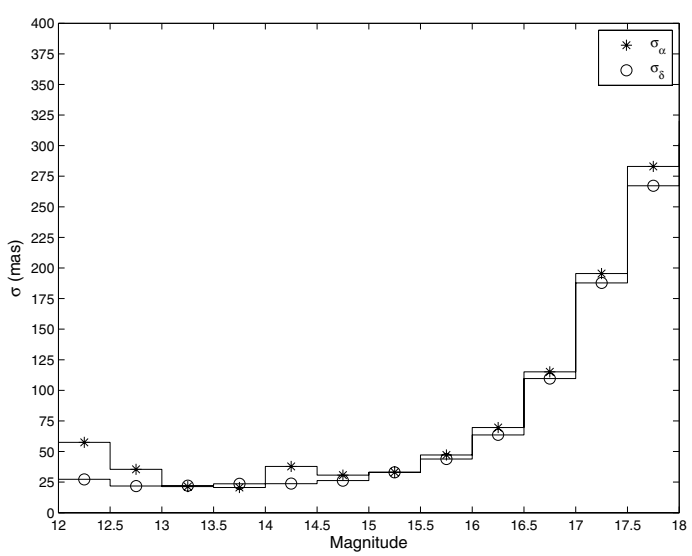

Figure 2. Precision per coordinate as a function of magnitude.

3rd-order model is suitable for reductions. The relationship between the residuals and magnitudes is plotted in Fig.1(b), where one point represents 5 reference stars. Apart from brighter stars ( $<13 \mathrm{mag}$ ), there is no obvious magnitude equation with respect to the reference stars.

Based on repeated observations, a histogram of the precision as a function of magnitude is shown in Fig. 2. For this group of CCD images, the astrometric precision for objects brighten than $16.5 \mathrm{mag}$ is better than 70 mas per coordinate.

\section{References}

Zacharias, N., et al. 2004, AJ, 127, 3043 